\title{
On a Coupled System of Shallow Water Equations Admitting Travelling Wave Solutions
}

\author{
D. Burini, ${ }^{1,2}$ S. De Lillo, ${ }^{1,2}$ and D. Skouteris ${ }^{3}$ \\ ${ }^{1}$ Dipartimento di Matematica e Informatica, Università degli Studi di Perugia, Via Vanvitelli 1, 06123 Perugia, Italy \\ ${ }^{2}$ Istituto Nazionale di Fisica Nucleare, Sezione di Perugia, 06123 Perugia, Italy \\ ${ }^{3}$ Scuola Normale Superiore, Piazza dei Cavalieri 7, 56126 Pisa, Italy \\ Correspondence should be addressed to S. De Lillo; silvana.delillo@pg.infn.it
}

Received 20 January 2015; Accepted 25 March 2015

Academic Editor: Robert A. Van Gorder

Copyright @ 2015 D. Burini et al. This is an open access article distributed under the Creative Commons Attribution License, which permits unrestricted use, distribution, and reproduction in any medium, provided the original work is properly cited.

\begin{abstract}
We consider three inviscid, incompressible, irrotational fluids that are contained between the rigid walls $y=-h_{1}$ and $y=h+H$ and that are separated by two free interfaces $\eta_{1}$ and $\eta_{2}$. A generalized nonlocal spectral (NSP) formulation is developed, from which asymptotic reductions of stratified fluids are obtained, including coupled nonlinear generalized Boussinesq equations and $(1+1)$ dimensional shallow water equations. A numerical investigation of the $(1+1)$-dimensional case shows the existence of solitary wave solutions which have been investigated for different values of the characteristic parameters.
\end{abstract}

\section{Introduction}

Since some early studies started in the 1950s (see $[1,2]$ and refs. therein), much interest has been devoted over the years to the flow of stratified fluids, both from a theoretical and from applied point of view. In particular, multifluid flows characterized by gravitationally stable density interfaces are a useful mathematical model in geophysics and engineering [3-5]. On the other hand, many analytical studies have been dedicated to the equations that describe the system of two ideal fluids, separated by a free interface [6]. Indeed, a great effort has been devoted to modeling the evolution of the internal waves in a two-fluid system and to deriving asymptotic reductions (see [7] for a recent review). Among them, of particular relevance are the Benjamin-Ono (BO) equation $[8,9]$ and the intermediate long wave (ILW) equation $[10,11]$. Moreover, in [12] model equations governing the evolution of fully nonlinear long waves at the interface of two immiscible ideal fluids were derived, while the weakly nonlinear limit was treated in [13]; finally in [14] a Hamiltonian formulation of the two-fluid system was derived and through a perturbative theory a systematic analysis of the long waves scaling regimes was carried out. On the other hand, in recent years, a nonlocal formulation of water waves for both $(1+1)$ and
$(2+1)$ dimensions was presented in [15], where the original equations with unknown boundary conditions are replaced by an integrodifferential equation and a nonlinear partial differential equation, both of which are formulated in a known domain. The nonlocal formulation obtained in [15] is derived from the general approach to studying boundary value problems for linear and nonlinear PDEs introduced in [16]. A crucial role in such approach is played by a nonlocal equation, called the global relation [16]. A generalization of the results obtained in [15] was presented in [17], where a nonlocal formulation was derived, governing two ideal fluids separated by a free interface and bounded above either by a rigid lid or by a free surface [17]. Due to the dependence on a free spectral parameter, the corresponding equations are usually called the nonlocal spectral (NSP) equations of the two-fluid system. The NSP equations were particularly useful for deriving asymptotic approximations; we wish to point out an asymptotically $(2+1)$-dimensional generalization of the intermediate long wave (ILW) equation reported in [17] which includes the KP equation and the Benjamin-Ono equation as limiting cases. Numerical investigations indicated the existence of lump type solutions, with a speed versus amplitude relationship shown to be linear in the shallow, intermediate, and deep water regime. 


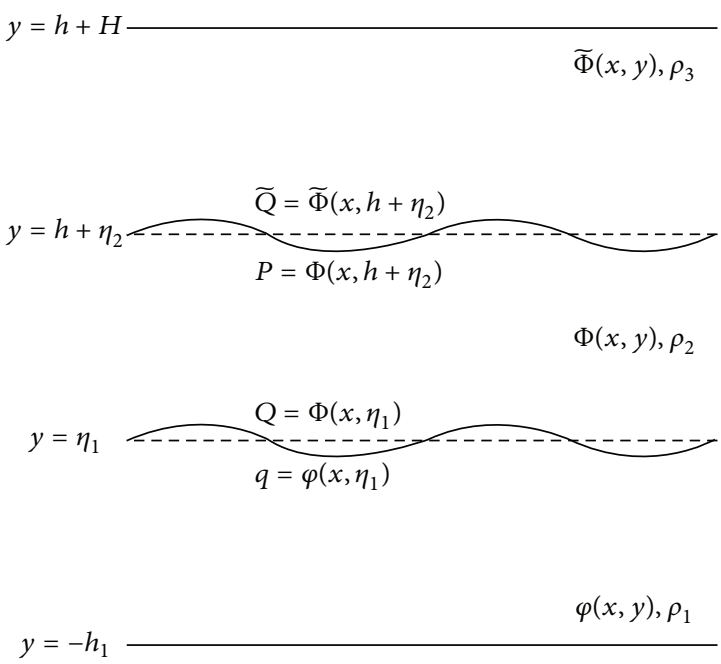

Figure 1: Three fluids with two free internal interfaces.

However, to the best of our knowledge, the phenomenological models for more than two fluids mentioned at the beginning of this section have not been paralleled by any analytical study. This prompted us to develop a generalization of the NSP formulation to the case of three ideal fluids, separated by two free interfaces and limited above by a rigid lid. Namely, we consider three inviscid, incompressible, irrotational fluids that are confined between the rigid lids $y=-h_{1}$ and $y=h+H$ and are separated by two free interfaces $\eta_{1}(x, t)$ and $\eta_{2}(x, t)$. We derive in the following the NSP equations governing the evolution of the three-fluid system. Specifically, define the functions $q(x, t)=\varphi\left(x, \eta_{1}(x, t), t\right)$, $Q(x, t)=\Phi\left(x, \eta_{1}(x, t), t\right), P(x, t)=\Phi\left(x, h+\eta_{2}(x, t), t\right)$ and $\widetilde{Q}(x, t)=\widetilde{\Phi}\left(x, h+\eta_{2}(x, t), t\right)$, where $\varphi(x, y), \Phi(x, y)$, and $\widetilde{\Phi}(x, y)$ are, respectively, the velocity potentials in the lower, the intermediate, and upper layer (see Figure 1).

The NSP formulation is given by the following equations.

First layer (bottom)

$$
\begin{aligned}
& \int_{\mathbb{R}^{2}} e^{i k x} \cosh \left(|k|\left(h_{1}+\eta_{1}\right)\right) \eta_{1 t} d x \\
& \quad=i \int_{\mathbb{R}^{2}} e^{i k x} \frac{\sinh \left(|k|\left(h_{1}+\eta_{1}\right)\right)}{|k|}(k \cdot \nabla) q d x,
\end{aligned}
$$

intermediate layer

$$
\begin{aligned}
\int_{\mathbb{R}^{2}} e^{i k x} \sinh \left(|k| \eta_{2}\right) \eta_{2 t} d x \\
\quad-\int_{\mathbb{R}^{2}} e^{i k x} \sinh \left(|k|\left(\eta_{1}-h\right)\right) \eta_{1 t} d x \\
=-i \int_{\mathbb{R}^{2}} e^{i k x} \frac{\cosh \left(|k|\left(\eta_{1}-h\right)\right)}{|k|}(k \cdot \nabla) Q d x \\
\quad+i \int_{\mathbb{R}^{2}} e^{i k x} \frac{\cosh \left(|k| \eta_{2}\right)}{|k|}(k \cdot \nabla) P d x,
\end{aligned}
$$

$$
\begin{aligned}
\int_{\mathbb{R}^{2}} e^{i k x} \sinh \left(|k|\left(\eta_{2}+h\right)\right) \eta_{2 t} d x \\
-\int_{\mathbb{R}^{2}} e^{i k x} \sinh \left(|k| \eta_{1}\right) \eta_{1 t} d x \\
=-i \int_{\mathbb{R}^{2}} e^{i k x} \frac{\cosh \left(|k| \eta_{1}\right)}{|k|}(k \cdot \nabla) Q d x \\
\quad+i \int_{\mathbb{R}^{2}} e^{i k x} \frac{\cosh \left(|k|\left(\eta_{2}+h\right)\right)}{|k|}(k \cdot \nabla) P d x,
\end{aligned}
$$

third layer (top)

$$
\begin{aligned}
& \int_{\mathbb{R}^{2}} e^{i k x} \cosh \left(|k|\left(\eta_{2}-H\right)\right) \eta_{2 t} d x \\
& \quad=i \int_{\mathbb{R}^{2}} e^{i k x} \frac{\sinh \left(|k|\left(\eta_{2}-H\right)\right)}{|k|}(k \cdot \nabla) \widetilde{Q} d x,
\end{aligned}
$$

\section{Bernoulli's equations}

$$
\begin{aligned}
& \rho_{1}\left(q_{t}\right.\left.+\frac{1}{2}|\nabla q|^{2}+g \eta_{1}-\frac{\left(\eta_{1 t}+\nabla q \cdot \nabla \eta_{1}\right)^{2}}{2\left(1+\left|\nabla \eta_{1}\right|^{2}\right)}\right) \\
&-\rho_{2}\left(Q_{t}+\frac{1}{2}|\nabla Q|^{2}+g \eta_{1}-\frac{\left(\eta_{1 t}+\nabla Q \cdot \nabla \eta_{1}\right)^{2}}{2\left(1+\left|\nabla \eta_{1}\right|^{2}\right)}\right) \\
&= \sigma_{1} \nabla \cdot\left(\frac{\nabla \eta_{1}}{\sqrt{1+\left|\nabla \eta_{1}\right|^{2}}}\right), \\
& \rho_{2}\left(P_{t}+\frac{1}{2}|\nabla P|^{2}+g \eta_{2}-\frac{\left(\eta_{2 t}+\nabla P \cdot \nabla \eta_{2}\right)^{2}}{2\left(1+\left|\nabla \eta_{2}\right|^{2}\right)}\right) \\
& \quad-\rho_{3}\left(\widetilde{Q} t+\frac{1}{2}|\nabla \widetilde{Q}|^{2}+g \eta_{2}-\frac{\left(\eta_{2 t}+\nabla \widetilde{Q} \cdot \nabla \eta_{2}\right)^{2}}{2\left(1+\left|\nabla \eta_{2}\right|^{2}\right)}\right) \\
&=\sigma_{2} \nabla \cdot\left(\frac{\nabla \eta_{2}}{\sqrt{1+\left|\nabla \eta_{2}\right|^{2}}}\right) .
\end{aligned}
$$

In (1)-(5), $x=\left(x_{1}, x_{2}\right), k=\left(k_{1}, k_{2}\right), k x \equiv k \cdot x$, where $k$ is a free spectral parameter and the constants $g, \sigma_{1}$, and $\sigma_{2}$ denote gravity, surface tension of $\eta_{1}(x, t)$, and surface tension of $\eta_{2}(x, t)$, respectively.

In the next section, we derive the above equations, starting from the classic equations governing three ideal fluids separated by two free interfaces bounded above and below by rigid lids. Moreover, we derive conservation laws and integral identities for the three-fluid system for the NSP formulation.

Section 3 is devoted to the derivation of the weakly nonlinear equations: after a suitable nondimensionalization of the variables, we obtain the reduction to a system of shallow water equations in the weakly nonlinear limit. 
In Section 4, under the assumption of maximal balance, we introduce travelling wave variables moving only to the right and we study only the $(1+1)$-dimensional case. In terms of the new variables, we finally obtain a system of coupled nonlinear shallow water equations which we study numerically in terms of the parameters entering the theory and that we show to admit solitary wave solutions.

\section{A Nonlocal Spectral Formulation of the Classic Three-Fluid Equations}

2.1. A Weak Formulation of the Classic Three-Fluid Equations. We recall the classic equations governing three ideal fluids separated by two free interfaces $\eta_{1}$ and $\eta_{2}$ and bounded above and below by rigid lids. It is assumed that the lower fluid is of density $\rho_{1}$, the intermediate fluid is of density $\rho_{2}$, and the upper fluid is of density $\rho_{3}$ with $\rho_{1}>\rho_{2}>\rho_{3}$. The equations are given in terms of the interface variables and the velocity potentials $\varphi, \Phi$, and $\widetilde{\Phi}$ associated with the lower, intermediate, and upper fluid domains, respectively,

$$
\begin{gathered}
\Delta \varphi=0, \quad \text { for }-h_{1}<y<\eta_{1}, \\
\left.\varphi_{y}\right|_{y=-h_{1}}=0, \\
\eta_{1 t}=\left.\left(\varphi_{y}-\nabla_{x} \varphi \cdot \nabla_{x} \eta_{1}\right)\right|_{y=\eta_{1}}, \\
\Delta \Phi=0, \quad \text { for } \eta_{1}<y<h+\eta_{2}, \\
\eta_{1 t}=\left.\left(\Phi_{y}-\nabla_{x} \Phi \cdot \nabla_{x} \eta_{1}\right)\right|_{y=\eta_{1}}, \\
\eta_{2 t}=\left.\left(\Phi_{y}-\nabla_{x} \Phi \cdot \nabla_{x} \eta_{2}\right)\right|_{y=h+\eta_{2}}, \\
\Delta \widetilde{\Phi}=0, \quad \text { for } h+\eta_{2}<y<H+h, \\
\eta_{2 t}=\left.\left(\widetilde{\Phi}_{y}-\nabla_{x} \widetilde{\Phi} \cdot \nabla_{x} \eta_{2}\right)\right|_{y=h+\eta_{2}}, \\
\rho_{1}\left(\varphi_{t}+\frac{1}{2}|\nabla \varphi|^{2}+g \eta_{1}\right)-\rho_{2}\left(\Phi_{t}+\frac{1}{2}|\nabla \Phi|^{2}+g \eta_{1}\right) \\
=\sigma_{1} \nabla \cdot\left(\frac{\nabla \eta_{1}}{\sqrt{1+\left|\nabla \eta_{1}\right|^{2}}}\right), \quad \text { on } y=\eta_{1}, \\
\rho_{2}\left(\Phi_{t}+\frac{1}{2}|\nabla \Phi|^{2}+g \eta_{2}\right)-\rho_{3}\left(\widetilde{\Phi}_{t}+\frac{1}{2}|\nabla \widetilde{\Phi}|^{2}+g \eta_{2}\right) \\
=\sigma_{2} \nabla \cdot\left(\frac{\nabla \eta_{2}}{\sqrt{1+\left|\nabla \eta_{2}\right|^{2}}}\right), \quad \text { on } y=h+\eta_{2} .
\end{gathered}
$$

We also require that $|\nabla \varphi| \rightarrow 0,|\nabla \Phi| \rightarrow 0$ and $|\nabla \widetilde{\Phi}| \rightarrow$ 0 as $|x| \rightarrow \infty$. In the previous equations, the constants $g, \sigma_{1}$, and $\sigma_{2}$ denote gravity, the surface tension associated with the free interface $\eta_{1}$, and the surface tension associated with the free surface $\eta_{2}$. Equations (6), (9), and (12) express the fact that fluids are divergence and curl-free. Equations (15)-(16) express that the jump in pressure across an interface is balanced by surface tension.

We now obtain a weak formulation of (6)-(16), expressed in terms of $\eta_{1}, \eta_{2}, q, Q, P$, and $\widetilde{Q}$. Let us define the domains

$$
\begin{gathered}
D\left(\eta_{1}\right) \equiv\left\{(x, y) \in \mathbb{R}^{2} \mid x \in \mathbb{R}^{2},-h_{1}<y<\eta_{1}(x, t)\right\}, \\
D\left(\eta_{1}, \eta_{2}\right) \equiv\left\{(x, y) \in \mathbb{R}^{2} \mid x \in \mathbb{R}^{2},\right. \\
\left.\eta_{1}(x, t)<y<h+\eta_{2}(x, t)\right\}, \\
D\left(\eta_{2}\right) \equiv\left\{(x, y) \in \mathbb{R}^{2} \mid x \in \mathbb{R}^{2},\right. \\
\left.h+\eta_{2}(x, t)<y<h+H\right\} .
\end{gathered}
$$

For any bounded, harmonic functions $\psi, \Psi, \widetilde{\Psi}$, defined in $D\left(\eta_{1}\right), D\left(\eta_{1}, \eta_{2}\right)$, and $D\left(\eta_{2}\right)$, respectively, and satisfying

$$
\begin{gathered}
\Delta \psi=0, \quad \text { for }(x, y) \in D\left(\eta_{1}\right), \\
\left.\psi_{y}\right|_{y=-h_{1}}=0, \\
\Delta \widetilde{\Psi}=0, \quad \text { for }(x, y) \in D\left(\eta_{2}\right), \\
\left.\widetilde{\Psi}_{y}\right|_{y=H+h}=0, \\
|\nabla \psi|,|\nabla \Psi|,|\nabla \widetilde{\Psi}| \longrightarrow 0, \quad \text { as }|x| \longrightarrow \infty,
\end{gathered}
$$

the following identities hold at any given time $t$ :

$$
\begin{aligned}
& \int_{\mathbb{R}^{2}}\left(\left.\psi\right|_{y=\eta_{1}}\right) \eta_{1 t} d x \\
& \quad-\left.\int_{\mathbb{R}^{2}} q\left(\psi_{y}-\nabla_{x} \psi \cdot \nabla_{x} \eta_{1}\right)\right|_{y=\eta_{1}} d x=0, \\
& \int_{\mathbb{R}^{2}}\left(\left.\Psi\right|_{y=h+\eta_{2}}\right) \eta_{2 t} d x-\int_{\mathbb{R}^{2}}\left(\left.\Psi\right|_{y=\eta_{1}}\right) \eta_{1 t} d x \\
& \quad+\left.\int_{\mathbb{R}^{2}} Q\left(\Psi_{y}-\nabla_{x} \Psi \cdot \nabla_{x} \eta_{1}\right)\right|_{y=\eta_{1}} d x \\
& \quad-\left.\int_{\mathbb{R}^{2}} P\left(\Psi_{y}-\nabla_{x} \Psi \cdot \nabla_{x} \eta_{2}\right)\right|_{y=h+\eta_{2}} d x=0, \\
& \int_{\mathbb{R}^{2}}\left(\left.\widetilde{\Psi}\right|_{y=h+\eta_{2}}\right) \eta_{2 t} d x \\
& \quad-\left.\int_{\mathbb{R}^{2}} \widetilde{Q}\left(\widetilde{\Psi}-\nabla_{x} \widetilde{\Psi} \cdot \nabla_{x} \eta_{2}\right)\right|_{y=h+\eta_{2}} d x=0 .
\end{aligned}
$$

Equations (19) and (21), together with the Bernoulli equations (15) and (16), constitute a nonlocal system of equations describing the three-fluid system.

2.2. Derivation of the Nonlocal Spectral Equations. We derive from (19) the NSP equation (1) given in the Introduction. To motivate the derivation, suppose that $\psi(x, y)$ satisfies

$$
\Delta \psi(x, y)=0, \quad \psi_{y}\left(x,-h_{1}\right)=0 .
$$


Define $\widehat{\psi}(k, y)$ by

$$
\widehat{\psi}(k, y)=\frac{1}{(2 \pi)^{2}} \int_{\mathbb{R}^{2}} e^{-i k x} \psi(x, y) d x
$$

Then, conditions (22) turn into the following:

$$
\widehat{\psi}_{y y}(k, y)=|k|^{2} \widehat{\psi}(k, y), \quad \widehat{\psi}_{y}(k,-y)=0 .
$$

The solution to the above ODE is given by

$$
\widehat{\psi}(k, y)=\widehat{\xi}(k) \cosh \left(|k|\left(y+h_{1}\right)\right)
$$

reverting back to physical space,

$$
\psi(x, y)=\int_{\mathbb{R}^{2}} e^{i k x} \widehat{\xi}(k) \cosh \left(|k|\left(y+h_{1}\right)\right) d k .
$$

The previous equation shows that any harmonic function $\psi$ whose $y$-derivative at $y=-h_{1}$ vanishes can formally be written as a sum of functions $e^{i k x} \cosh \left(|k|\left(y+h_{1}\right)\right)$. Therefore, by linearity it suffices to require that (19) holds for the parametrized family of functions

$$
\psi_{k}(x, y)=e^{i k x} \cosh \left(|k|\left(y+h_{1}\right)\right)
$$

Substituting $\psi_{k}(x, y)$ into (19), we obtain

$$
\begin{aligned}
& \int_{\mathbb{R}^{2}} e^{i k x} \cosh \left(|k|\left(\eta_{1}+h_{1}\right)\right) \eta_{1 t} d x \\
&=\int_{\mathbb{R}^{2}} q\left(e ^ { i k x } \left(|k| \sinh \left(|k|\left(\eta_{1}+h_{1}\right)\right)\right.\right. \\
&\left.\left.-i \cosh \left(|k|\left(\eta_{1}+h_{1}\right)\right)(k \cdot \nabla) \eta_{1}\right)\right) d x .
\end{aligned}
$$

We simplify (28) by noting that

$$
\begin{aligned}
|k| e^{i k x} \sinh \left(|k|\left(\eta_{1}+h_{1}\right)\right) & \\
& -i e^{i k x} \cosh \left(|k|\left(\eta_{1}+h_{1}\right)\right)(k \cdot \nabla) \eta_{1} \\
= & -i \nabla \cdot\left(\frac{k}{|k|} \sinh \left(|k|\left(\eta_{1}+h_{1}\right) e^{i k x}\right)\right) .
\end{aligned}
$$

Using this identity in (28) and integrating by parts, we finally get the nonlocal spectral equation (1) as follows:

$$
\begin{aligned}
\int_{\mathbb{R}^{2}} e^{i k x} \cosh \left(|k|\left(\eta_{1}+h_{1}\right)\right) \eta_{1 t} d x \\
\quad=i \int_{\mathbb{R}^{2}} e^{i k x} \frac{\sinh \left(|k|\left(\eta_{1}+h_{1}\right)\right)}{|k|}(k \cdot \nabla) q d x .
\end{aligned}
$$

Along the same lines used for the first layer, let us derive (2)-(3) from (20).

We define the basic functions

$$
\Psi_{k}(x, y)=e^{i k x} \sinh (|k|(y-h))
$$

and putting $\Psi_{k}$ into (20) gives

$$
\begin{aligned}
& \int_{\mathbb{R}^{2}} e^{i k x} \sinh \left(|k| \eta_{2}\right) \eta_{2 t} d x \\
& \quad-\int_{\mathbb{R}^{2}} e^{i k x} \sinh \left(|k|\left(\eta_{1}-h\right)\right) \eta_{1 t} d x \\
& =-\int_{\mathbb{R}^{2}} e^{i k x} Q\left[|k| \cosh \left(|k|\left(\eta_{1}-h\right)\right)\right. \\
& \left.\quad-i \sinh \left(|k|\left(\eta_{1}-h\right)\right)(k \cdot \nabla) \eta_{1}\right] d x \\
& +\int_{\mathbb{R}^{2}} e^{i k x} P\left[|k| \cosh \left(|k| \eta_{2}\right)\right. \\
& \left.-i \sinh \left(|k| \eta_{2}\right)(k \cdot \nabla) \eta_{2}\right] d x .
\end{aligned}
$$

We simplify (32) by noting that

$$
\begin{aligned}
& e^{i k x} {\left[|k| \cosh \left(|k|\left(\eta_{1}-h\right)\right)\right.} \\
&\left.-i \sinh \left(|k|\left(\eta_{1}-h\right)\right)(k \cdot \nabla) \eta_{1}\right] \\
&=-i \nabla \cdot\left(\frac{k}{|k|} \cosh \left(|k|\left(\eta_{1}-h\right)\right) e^{i k x}\right), \\
& e^{i k x}\left[|k| \cosh \left(|k| \eta_{2}\right)-i \sinh \left(|k| \eta_{2}\right)(k \cdot \nabla) \eta_{2}\right] \\
&=-i \nabla \cdot\left(\frac{k}{|k|} \cosh \left(|k| \eta_{2}\right) e^{i k x}\right) .
\end{aligned}
$$

Using these identities in (32) and integrating by parts, we finally get the nonlocal spectral equation (2).

Similarly, let us consider the basic functions

$$
\Psi_{k}(x, y)=e^{i k x} \sinh (|k| y)
$$

and put $\Psi_{k}$ into (20), obtaining

$$
\begin{aligned}
& \int_{\mathbb{R}^{2}} e^{i k x} \sinh \left(|k|\left(\eta_{2}+h\right)\right) \eta_{2 t} d x \\
& \quad-\int_{\mathbb{R}^{2}} e^{i k x} \sinh \left(|k| \eta_{1}\right) \eta_{1 t} d x \\
& =-\int_{\mathbb{R}^{2}} e^{i k x} \mathrm{Q}\left[|k| \cosh \left(|k| \eta_{1}\right)\right. \\
& \left.-i \sinh \left(|k| \eta_{1}\right)(k \cdot \nabla) \eta_{1}\right] d x \\
& +\int_{\mathbb{R}^{2}} e^{i k x} P\left[|k| \cosh \left(|k|\left(\eta_{2}+h\right)\right)\right. \\
& \left.-i \sinh \left(|k|\left(\eta_{2}+h\right)\right)(k \cdot \nabla) \eta_{2}\right] d x .
\end{aligned}
$$


We simplify (35) by noting that

$$
\begin{aligned}
e^{i k x} & {\left[|k| \cosh \left(|k| \eta_{1}\right)-i \sinh \left(|k| \eta_{1}\right)(k \cdot \nabla) \eta_{1}\right] } \\
& =-i \nabla \cdot\left(\frac{k}{|k|} \cosh \left(|k| \eta_{1}\right) e^{i k x}\right), \\
e^{i k x} & {\left[|k| \cosh \left(|k|\left(\eta_{2}+h\right)\right)-i \sinh \left(|k|\left(\eta_{2}+h\right)\right)(k \cdot \nabla) \eta_{2}\right] } \\
& =-i \nabla \cdot\left(\frac{k}{|k|} \cosh \left(|k|\left(\eta_{2}+h\right)\right) e^{i k x}\right) .
\end{aligned}
$$

Using these identities in (35) and integrating by parts, we finally get the nonlocal spectral equation (3).

For the top layer, we require that (21) holds for the parametrized family of functions

$$
\widetilde{\Psi}_{k}(x, y)=e^{i k x} \cosh (|k|(y-(H+h))) .
$$

Substituting $\widetilde{\Psi}_{k}(x, y)$ into (21), we obtain

$$
\begin{aligned}
& \int_{\mathbb{R}^{2}} e^{i k x} \cosh \left(|k|\left(\eta_{2}-H\right)\right) \eta_{2 t} d x \\
&=\int_{\mathbb{R}^{2}} \widetilde{Q}\left(e ^ { i k x } \left(|k| \sinh \left(|k|\left(\eta_{2}-H\right)\right)\right.\right. \\
&\left.\left.-i \cosh \left(|k|\left(\eta_{2}-H\right)\right)(k \cdot \nabla) \eta_{2}\right)\right) d x
\end{aligned}
$$

We simplify (38) by noting that

$$
\begin{aligned}
|k| e^{i k x} \sinh \left(|k|\left(\eta_{2}-H\right)\right) & \\
& -i e^{i k x} \cosh \left(|k|\left(\eta_{2}-H\right)\right)(k \cdot \nabla) \eta_{2} \\
= & -i \nabla \cdot\left(\frac{k}{|k|} \sinh \left(|k|\left(\eta_{2}-H\right) e^{i k x}\right)\right) .
\end{aligned}
$$

Using this identity in (28) and integrating by parts, we finally get the nonlocal spectral equation (4).

2.3. Conservation Laws and Integral Identities. In [17], conservation laws and integral identities for a two-fluid system were derived from the nonlocal spectral formulation. We now derive the analogous conservation laws for the three-fluid system for the NSP formulation. We start by expanding (2), (3) for small $k$. At first order, by setting to zero the coefficient of $k$ and putting $k_{2}=0$, we get

$$
\begin{aligned}
& \int_{\mathbb{R}^{2}}\left(\eta_{2} \eta_{2 t}-\eta_{1} \eta_{1 t}+H \eta_{1 t}\right) d x=\int_{\mathbb{R}^{2}} x_{1}\left(Q_{x_{1}}-P_{x_{1}}\right) d x \\
& \int_{\mathbb{R}^{2}}\left(\eta_{2} \eta_{2 t}-\eta_{1} \eta_{1 t}+H \eta_{2 t}\right) d x=\int_{\mathbb{R}^{2}} x_{1}\left(Q_{x_{1}}-P_{x_{1}}\right) d x
\end{aligned}
$$

Subtracting (41) from (40), we obtain the result

$$
\partial_{t} \int_{\mathbb{R}^{2}}\left(\eta_{1}-\eta_{2}\right) d x=0
$$

which corresponds to mass conservation in the intermediate fluid domain.

Similarly, by setting to zero the coefficient of $k^{2}$, with $k_{2}=$ 0 , we get

$$
\begin{aligned}
& \partial_{t} \int_{\mathbb{R}^{2}} x_{1}\left(\frac{\eta_{2}^{2}}{2 H}-\frac{\eta_{1}^{2}}{2 H}+\eta_{1}\right) d x \\
& \quad=\int_{\mathbb{R}^{2}}\left[\frac{\eta_{2}^{2}}{2 H} P_{x_{1}}-\left(\frac{\eta_{1}^{2}}{2 H}+\frac{H}{2}-\eta_{1}\right) Q_{x_{1}}\right] d x, \\
& \partial_{t} \int_{\mathbb{R}^{2}} x_{1}\left(\frac{\eta_{2}^{2}}{2 H}-\frac{\eta_{1}^{2}}{2 H}+\eta_{2}\right) d x \\
& \quad=\int_{\mathbb{R}^{2}}\left[\left(\frac{\eta_{2}^{2}}{2 H}+\frac{H}{2}+\eta_{2}\right) P_{x_{1}}-\frac{\eta_{1}^{2}}{2 H} Q_{x_{1}}\right] d x .
\end{aligned}
$$

When we subtract (43) from (44), we obtain

$$
\begin{aligned}
& \partial_{t} \int_{\mathbb{R}^{2}} x_{1}\left(\eta_{2}-\eta_{1}\right) d x \\
& \quad=\int_{\mathbb{R}^{2}}\left[\left(\frac{H}{2}+\eta_{2}\right) P_{x_{1}}+\left(\frac{H}{2}-\eta_{1}\right) Q_{x_{1}}\right] d x
\end{aligned}
$$

similarly, if we set $k_{1}=0$, at the same order we get the corresponding equation:

$$
\begin{aligned}
& \partial_{t} \int_{\mathbb{R}^{2}} x_{1}\left(\eta_{2}-\eta_{1}\right) d x \\
& \quad=\int_{\mathbb{R}^{2}}\left[\left(\frac{H}{2}+\eta_{2}\right) P_{x_{2}}+\left(\frac{H}{2}-\eta_{1}\right) Q_{x_{2}}\right] d x .
\end{aligned}
$$

Equations (45) and (46) describe the evolution of the center of mass; the right-hand side is the momentum of the fluid.

Next, we obtain a last identity at third order, setting to zero the coefficient of $k^{3}$ :

$$
\begin{gathered}
\partial_{t} \int_{\mathbb{R}^{2}}\left[\frac{x_{j}}{2}\left(\eta_{2}-\eta_{1}\right)-\frac{H}{4}\left(\eta_{2}^{2}+\eta_{1}^{2}\right)-\frac{1}{6}\left(\eta_{2}^{3}+\eta_{1}^{3}\right)\right] d x \\
=\int_{\mathbb{R}^{2}} x_{j}\left[\left(\frac{H}{2}+\eta_{2}\right) P_{x_{j}}+\left(\frac{H}{2}-\eta_{1}\right) Q_{x_{j}}\right] d x,
\end{gathered}
$$

with $j=1,2$; the above is a virial type formula, analogous to the one obtained in [17].

\section{Weakly Nonlinear Equations}

3.1. Nondimensionalization of the NSP Equations. In order to derive weakly nonlinear equations, we nondimensionalize all physical variables in (1)-(5) according to

$$
\begin{gathered}
x_{1}^{\prime}=\frac{x_{1}}{L}, \quad x_{2}^{\prime}=\gamma \frac{x_{2}}{L}, \quad t^{\prime}=\frac{c_{0}}{L} t, \\
\eta_{1,2}=a \eta_{1,2}^{\prime}, \quad \beta=a \beta^{\prime},
\end{gathered}
$$




$$
\begin{array}{ll}
q=\frac{a g L}{c_{0}} q^{\prime}, & Q=\frac{a g H}{c_{0}} Q^{\prime}, \\
P=\frac{a g H}{c_{0}} P^{\prime}, & \widetilde{Q}=\frac{a g L}{c_{0}} \widetilde{Q}^{\prime},
\end{array}
$$

where $L$ is a characteristic wavelength, $\gamma$ is a nondimensional parameter, and $a$ and $c_{0}=\sqrt{g H}$ are a characteristic amplitude and velocity, respectively.

Then, (1)-(5) become, after dropping primes and letting $k \rightarrow-k$,

$$
\begin{aligned}
& \text { first layer (bottom) } \\
& \int_{\mathbb{R}^{2}} e^{-i k x} \cosh \left(|k|\left(\alpha_{1}+\varepsilon \mu \eta_{1}\right)\right) \eta_{1 t} d x \\
& =\frac{i}{\mu} \int_{\mathbb{R}^{2}} e^{-i k x} \frac{\sinh \left(|k|\left(\alpha_{1}+\varepsilon \mu \eta_{1}\right)\right)}{|k|}(k \cdot \nabla) q d x, \\
& \text { intermediate layer } \\
& \int_{\mathbb{R}^{2}} e^{-i k x} \sinh \left(|k| \varepsilon \mu \eta_{2}\right) \eta_{2 t} d x \\
& -\int_{\mathbb{R}^{2}} e^{-i k x} \sinh \left(|k|\left(\varepsilon \mu \eta_{1}-\alpha_{2}\right)\right) \eta_{1 t} d x \\
& =-i \int_{\mathbb{R}^{2}} e^{-i k x} \frac{\cosh \left(|k|\left(\varepsilon \mu \eta_{1}-\alpha_{2}\right)\right)}{|k|}(k \cdot \nabla) Q d x \\
& +i \int_{\mathbb{R}^{2}} e^{-i k x} \frac{\cosh \left(|k| \varepsilon \mu \eta_{2}\right)}{|k|}(k \cdot \nabla) P d x, \\
& \int_{\mathbb{R}^{2}} e^{-i k x} \sinh \left(|k|\left(\alpha_{2}+\varepsilon \mu \eta_{2}\right)\right) \eta_{2 t} d x \\
& -\int_{\mathbb{R}^{2}} e^{-i k x} \sinh \left(|k| \varepsilon \mu \eta_{1}\right) \eta_{1 t} d x \\
& =-i \int_{\mathbb{R}^{2}} e^{-i k x} \frac{\cosh \left(|k| \varepsilon \mu \eta_{1}\right)}{|k|}(k \cdot \nabla) Q d x \\
& +i \int_{\mathbb{R}^{2}} e^{-i k x} \frac{\cosh \left(|k|\left(\alpha_{2}+\varepsilon \mu \eta_{2}\right)\right)}{|k|}(k \cdot \nabla) P d x,
\end{aligned}
$$

third layer (top)

$$
\begin{aligned}
& \int_{\mathbb{R}^{2}} e^{-i k x} \cosh \left(|k|\left(\varepsilon \mu \eta_{2}-\mu\right)\right) \eta_{2 t} d x \\
& =\frac{i}{\mu} \int_{\mathbb{R}^{2}} e^{-i k x} \frac{\sinh \left(|k|\left(\varepsilon \mu \eta_{2}-\mu\right)\right)}{|k|}(k \cdot \nabla) \widetilde{Q} d x,
\end{aligned}
$$

\section{Bernoulli's equations}

$$
\begin{aligned}
& q_{t}+\frac{1}{2} \varepsilon|\nabla q|^{2}+\eta_{1}-\varepsilon \mu^{2} \frac{\left(\eta_{1 t}+\varepsilon \nabla q \nabla \eta_{1}\right)^{2}}{2\left(1+\varepsilon^{2} \mu^{2}\left|\nabla \eta_{1}\right|^{2}\right)} \\
& -\tilde{\rho}\left[\mu Q_{t}+\frac{1}{2} \varepsilon \mu^{2}|\nabla Q|^{2}+\eta_{1}\right. \\
& \left.-\varepsilon \mu^{2} \frac{\left(\eta_{1 t}+\varepsilon \mu \nabla Q \nabla \eta_{1}\right)^{2}}{2\left(1+\varepsilon^{2} \mu^{2}\left|\nabla \eta_{1}\right|^{2}\right)}\right] \\
& =\mu^{2} \widetilde{\sigma}_{1} \nabla\left[\frac{\nabla \eta_{1}}{\sqrt{1+\varepsilon^{2} \mu^{2}\left|\nabla \eta_{1}\right|^{2}}}\right] \text {, } \\
& \mu P_{t}+\frac{1}{2} \varepsilon \mu^{2}|\nabla P|^{2}+\eta_{2}-\varepsilon \mu^{2} \frac{\left(\eta_{2 t}+\varepsilon \mu \nabla P \nabla \eta_{2}\right)^{2}}{2\left(1+\varepsilon^{2} \mu^{2}\left|\nabla \eta_{2}\right|^{2}\right)} \\
& -\rho\left[\widetilde{Q}_{t}+\frac{1}{2} \varepsilon|\nabla \widetilde{Q}|^{2}+\eta_{2}-\varepsilon \mu^{2} \frac{\left(\eta_{2 t}+\varepsilon \nabla \widetilde{Q} \nabla \eta_{2}\right)^{2}}{2\left(1+\varepsilon^{2} \mu^{2}\left|\nabla \eta_{2}\right|^{2}\right)}\right] \\
& =\mu^{2} \widetilde{\sigma}_{2} \nabla\left[\frac{\nabla \eta_{2}}{\sqrt{1+\varepsilon^{2} \mu^{2}\left|\nabla \eta_{2}\right|^{2}}}\right] \text {. }
\end{aligned}
$$

In the above relations, $\varepsilon=a / H$ is the nonlinearity ratio and $\mu=H / L$ is the so-called aspect ratio [12]; moreover, it is

$$
\begin{aligned}
& \alpha_{1}=\frac{h_{1}}{L}, \quad \alpha_{2}=\frac{h}{L}, \quad \tilde{\sigma}_{1}=\frac{\sigma_{1}}{H^{2} g \rho_{1}}, \\
& \tilde{\sigma}_{2}=\frac{\sigma_{2}}{H^{2} g \rho_{2}}, \quad \tilde{\rho}=\frac{\rho_{2}}{\rho_{1}}, \quad \rho=\frac{\rho_{3}}{\rho_{2}} .
\end{aligned}
$$

In the following, we assume $\alpha_{j} \ll 1, j=1,2$ and $\mu \ll 1$, which corresponds to the case of long waves; moreover, we take the nonlinearity ratio $\varepsilon$ to be $\varepsilon=O(\mu)$.

The above assumptions imply that we are interested to derive asymptotic reductions of the NSP equations in the case of weakly nonlinear long waves.

3.2. Derivation of the Equations. We first expand (49) and (53) in $\mu$ and $\gamma^{2}$. From (49), taking the Inverse Fourier Transform (IFT), we get

$$
q_{x_{1}}=i \mu \operatorname{coth}\left(\alpha_{1} D_{1}\right) \eta_{1 t}+o\left(\mu^{2}\right),
$$

while (53) gives

$$
\begin{aligned}
\eta_{1}= & -\frac{q_{t}}{(1-\tilde{\rho})}-\frac{\varepsilon}{2(1-\tilde{\rho})}\left(q_{x_{1}}+\gamma^{2} q_{x_{2}}\right)^{2} \\
& +\mu \frac{\tilde{\rho}}{(1-\tilde{\rho})} Q_{t}+\mu^{2} \widetilde{\sigma}_{1} \eta_{1 x_{1} x_{1}}+o\left(\varepsilon \mu^{2}\right) .
\end{aligned}
$$


On the other hand, from (49), it is also useful to derive the following reduction:

$$
\begin{aligned}
\eta_{1 t}= & -\frac{\alpha_{1}}{\mu}\left(q_{x_{1} x_{1}}+\gamma^{2} q_{x_{2} x_{2}}\right) \\
& -\varepsilon \partial_{x_{1}}\left(\eta_{1} q_{x_{1}}\right)+o\left(\varepsilon \mu^{2}\right) .
\end{aligned}
$$

We now use (58) at leading order (L.O.) in (56) and, after some manipulations, we obtain from (57) the following equation:

$$
\begin{aligned}
\eta_{1 t}= & i \alpha_{1} \frac{\operatorname{coth}\left(\alpha_{1} D_{1}\right)}{(1-\tilde{\rho})} q_{x_{1} t t} \\
& -\frac{\varepsilon}{2(1-\tilde{\rho})} \partial_{t}\left(q_{x_{1}}+\gamma^{2} q_{x_{2}}\right)^{2} \\
& +\mu \frac{\tilde{\rho}}{(1-\tilde{\rho})} Q_{t t}+o\left(\mu^{2}\right) .
\end{aligned}
$$

By (58) and (59), the first generalized Boussinesq equation obtains

$$
\begin{aligned}
& \alpha_{1}\left(q_{x_{1} x_{1}}+\gamma^{2} q_{x_{2} x_{2}}\right)+i \mu \alpha_{1} \frac{\operatorname{coth}\left(\alpha_{1} D_{1}\right)}{(1-\tilde{\rho})} q_{x_{1} t t} \\
& -\frac{\varepsilon \mu}{(1-\tilde{\rho})} \partial_{x_{1}}\left(q_{t} q_{x_{1}}\right) \\
& -\frac{\varepsilon \mu}{2(1-\tilde{\rho})} \partial_{t}\left(q_{x_{1}}\right)^{2}+\mu^{2} \frac{\tilde{\rho}}{(1-\tilde{\rho})} Q_{t t} \\
& +o\left(\varepsilon \mu^{2}, \mu^{3}\right)=0 .
\end{aligned}
$$

We now turn our attention to the intermediate layer.

By equating the expansions of (50) and (51), we get the following expression for $Q_{x_{1}}$ :

$$
Q_{x_{1}}=-i \frac{\eta_{2 t}}{\sinh \left(\alpha_{2} D_{1}\right)}+i \operatorname{coth}\left(\alpha_{2} D_{1}\right) \eta_{1 t}+o(\varepsilon \mu)
$$

which in turn gives

$$
Q_{x_{1} t}=-i \frac{\eta_{2 t t}}{\sinh \left(\alpha_{2} D_{1}\right)}+i \operatorname{coth}\left(\alpha_{2} D_{1}\right) \eta_{1 t t}+o(\varepsilon \mu),
$$

where, from (58) and (69) at L.O., we have

$$
\eta_{1 t t}=-\frac{\alpha_{1}}{\mu} q_{x_{1} x_{1} t}, \quad \eta_{2 t t}=\widetilde{Q}_{x_{1} x_{1} t}
$$

When (63) are used in (62), after integrating with respect to $x_{1}$, we finally get

$$
Q_{t}=-i \frac{\widetilde{Q}_{x_{1} t}}{\sinh \left(\alpha_{2} D_{1}\right)}-i \frac{\alpha_{1}}{\mu} \operatorname{coth}\left(\alpha_{2} D_{1}\right) q_{x_{1} t}+o(\varepsilon \mu)
$$

We now take the $t$-derivative of (57) and use (64), obtaining

$$
\begin{aligned}
\eta_{1 t}= & -\frac{q_{t t}}{(1-\tilde{\rho})}+i \alpha_{1} \frac{\tilde{\rho}}{(1-\tilde{\rho})} \operatorname{coth}\left(\alpha_{2} D_{1}\right) q_{x_{1} t t} \\
& -\frac{\varepsilon}{2(1-\tilde{\rho})} \partial_{t}\left(q_{x_{1}}+\gamma^{2} q_{x_{2}}\right)^{2} \\
& -i \mu \frac{\tilde{\rho}}{(1-\tilde{\rho})} \frac{\widetilde{Q}_{x_{1} t t}}{\sinh \left(\alpha_{2} D_{1}\right)}+o\left(\varepsilon \mu^{2}\right) ;
\end{aligned}
$$

by equating (65) and (58), we get the second generalized Boussinesq equation:

$$
\begin{aligned}
& \alpha_{1}\left(q_{x_{1} x_{1}}+\gamma^{2} q_{x_{2} x_{2}}\right)-\frac{\mu}{(1-\tilde{\rho})} q_{t t} \\
& -\frac{\varepsilon \mu}{(1-\tilde{\rho})} \partial_{x_{1}}\left(q_{t} q_{x_{1}}\right)-\frac{\varepsilon \mu}{2(1-\tilde{\rho})} \partial_{t}\left(q_{x_{1}}\right)^{2} \\
& -i \alpha_{1} \mu \frac{\tilde{\rho}}{(1-\tilde{\rho})} \operatorname{coth}\left(\alpha_{2} D_{1}\right) q_{x_{1} t t} \\
& -i \mu^{2} \frac{\tilde{\rho}}{(1-\tilde{\rho})} \frac{\widetilde{Q}_{x_{1} t t}}{\sinh \left(\alpha_{2} D_{1}\right)}+o\left(\varepsilon \mu^{2}\right)=0 .
\end{aligned}
$$

We now expand (52) and take the IFT, getting

$$
\widetilde{Q}_{x_{1}}=-i \mu \operatorname{coth}\left(\mu D_{1}\right) \eta_{2 t}+o\left(\mu^{2}\right) \text {, }
$$

while from the expansion of (54) we get

$$
\begin{aligned}
\eta_{2}= & \frac{\rho}{(1-\rho)} \widetilde{Q}_{t}-\frac{\mu}{(1-\rho)} P_{t} \\
& +\frac{\varepsilon}{2} \frac{\rho}{(1-\rho)}\left(\widetilde{Q}_{x_{1}}+\gamma^{2} \widetilde{Q}_{x_{2}}\right)^{2}+o\left(\varepsilon \mu^{2}\right) .
\end{aligned}
$$

On the other hand, from (52), it is also useful to derive the following expansion:

$$
\eta_{2 t}=\widetilde{Q}_{x_{1} x_{1}}+\gamma^{2} \widetilde{Q}_{x_{2} x_{2}}-\varepsilon \partial_{x_{1}}\left(\eta_{2} \widetilde{Q}_{x_{1}}\right)+o(\varepsilon \mu) .
$$

We use (68) at L.O. in (69) and get

$$
\begin{aligned}
\eta_{2 t}= & \widetilde{Q}_{x_{1} x_{1}}+\gamma^{2} \widetilde{Q}_{x_{2} x_{2}} \\
& -\varepsilon \frac{\rho}{(1-\rho)} \partial_{x_{1}}\left(\widetilde{Q}_{t} \widetilde{Q}_{x_{1}}\right)+o(\varepsilon \mu) .
\end{aligned}
$$

In order to get an expression for $P_{t}$ in (68), we now expand (50), getting

$$
P_{x_{1}}=i \sinh \left(\alpha_{2} D_{1}\right) \eta_{1 t}+\cosh \left(\alpha_{2} D_{1}\right) Q_{x_{1}}+o(\varepsilon \mu) .
$$

By taking the $t$-derivative of (71) and integrating with respect to $x_{1}$, we get

$$
P_{t}=-i \frac{\alpha_{1}}{\mu} \sinh \left(\alpha_{2} D_{1}\right) q_{x_{1} t}+\cosh \left(\alpha_{2} D_{1}\right) Q_{t}+o(\varepsilon \mu)
$$

where the L.O. of (58) has also been used. 
When we substitute (72) back into (68) and take the $t$ derivative, we obtain

$$
\begin{aligned}
\eta_{2 t}= & i \frac{\alpha_{1}}{(1-\rho)} \sinh \left(\alpha_{2} D_{1}\right) q_{x_{1} t t} \\
& +\frac{\rho}{(1-\rho)} \widetilde{Q}_{t t}-\frac{\mu}{(1-\rho)} \cosh \left(\alpha_{2} D_{1}\right) Q_{t t} \\
& +\frac{\varepsilon}{2} \frac{\rho}{(1-\rho)} \partial_{t}\left(\widetilde{Q}_{x_{1}}\right)^{2}+o\left(\varepsilon \mu^{2}\right)=0 .
\end{aligned}
$$

By equating (70) and (73), we finally obtain the third generalized Boussinesq equation:

$$
\begin{aligned}
\widetilde{Q}_{t t}- & \frac{(1-\rho)}{\rho}\left(\widetilde{Q}_{x_{1} x_{1}}+\gamma^{2} \widetilde{Q}_{x_{2} x_{2}}\right) \\
& +i \frac{\alpha}{\rho} \sinh \left(\alpha_{2} D_{1}\right) q_{x_{1} t t} \\
& +\frac{\varepsilon}{2} \partial_{t}\left(\widetilde{Q}_{x_{1}}\right)^{2}-\frac{\mu}{\rho} \cosh \left(\alpha_{2} D_{1}\right) Q_{t t} \\
& +\varepsilon \partial_{x_{1}}\left(\widetilde{Q}_{t} \widetilde{Q}_{x_{1}}\right)+o(\varepsilon \mu)=0 .
\end{aligned}
$$

The system of 3 generalized Boussinesq equations (60), (66), and (74) will now be reduced to a system of 2 independent equations. We first take the shallow water limit, given by $\alpha_{1} \ll 1$ in (60) and $\alpha_{2} \ll 1$ in (66), obtaining the following reductions:

$$
\begin{aligned}
q_{x_{1} x_{1}} & +\gamma^{2} q_{x_{2} x_{2}}-\frac{\mu}{\alpha_{1}} \frac{1}{(1-\tilde{\rho})} q_{t t} \\
& -\frac{\varepsilon \mu}{\alpha_{1}} \frac{1}{(1-\tilde{\rho})} \partial_{x_{1}}\left(q_{t} q_{x_{1}}\right) \\
& -\frac{\varepsilon \mu}{\alpha_{1}} \frac{1}{2(1-\tilde{\rho})} \partial_{t}\left(q_{x_{1}}\right)^{2} \\
& +\frac{\mu^{2}}{\alpha_{1}} \frac{\tilde{\rho}}{(1-\tilde{\rho})} Q_{t t}+o\left(\varepsilon \mu^{2}\right)=0, \\
q_{x_{1} x_{1}} & +\gamma^{2} q_{x_{2} x_{2}}-\frac{\mu}{\alpha_{1}} \frac{1}{(1-\tilde{\rho})^{2}} q_{t t} \\
& -\frac{\varepsilon \mu}{\alpha_{1}} \frac{1}{(1-\tilde{\rho})} \partial_{x_{1}}\left(q_{t} q_{x_{1}}\right) \\
& -\frac{\varepsilon \mu}{\alpha_{1}} \frac{1}{2(1-\tilde{\rho})} \partial_{t}\left(q_{x_{1}}\right)^{2}-\frac{\mu}{\alpha_{2}} \frac{\tilde{\rho}}{(1-\tilde{\rho})} q_{t t} \\
& -\frac{\mu^{2}}{\alpha_{2} \alpha_{1}} \frac{\tilde{\rho}}{(1-\tilde{\rho})} \widetilde{Q_{t t}}+o\left(\varepsilon \mu^{2}\right)=0 .
\end{aligned}
$$

Next, the combination of (75) and (76) gives

$$
Q_{t t}=-\frac{\alpha_{1}}{\alpha_{2}} \frac{1}{\mu} q_{t t}-\frac{1}{\alpha_{2}} \widetilde{Q}_{t t}
$$

which we substitute back into (74) and we obtain

$$
\begin{aligned}
\widetilde{Q}_{t t} & -\frac{(1-\rho)}{\rho}\left(\widetilde{Q}_{x_{1} x_{1}}+\gamma^{2} \widetilde{Q}_{x_{2} x_{2}}\right) \\
= & \frac{\alpha_{1} \alpha_{2}}{\rho} q_{x_{1} x_{1} t t}-\frac{\varepsilon}{2} \partial_{t}\left(\widetilde{Q}_{x_{1}}\right)^{2} \\
& -\frac{\alpha_{1}}{\alpha_{2}} \frac{1}{\rho} q_{t t}-\frac{\mu}{\alpha_{2}} \frac{1}{\rho} \widetilde{Q}_{t t}-\varepsilon \partial_{x_{1}}\left(\widetilde{Q}_{t} \widetilde{Q}_{x_{1}}\right) .
\end{aligned}
$$

Rearranging (66), we get

$$
\begin{aligned}
q_{t t} & -\frac{\alpha_{1}}{\mu}(1-\tilde{\rho})\left(q_{x_{1} x_{1}}+\gamma^{2} q_{x_{2} x_{2}}\right) \\
= & \frac{\alpha_{1}}{\alpha_{2}} \widetilde{\rho} q_{t t} \\
& -\varepsilon \partial_{x_{1}}\left(q_{t} q_{x_{1}}\right)-\frac{\varepsilon}{2} \partial_{t}\left(q_{x_{1}}\right)^{2}+\frac{\mu}{\alpha_{2}} \widetilde{\rho} \widetilde{Q}_{t t} ;
\end{aligned}
$$

then the system of weakly nonlinear, shallow water equations is given by (78) and (79).

\section{Multiple Scale Derivation: Special Case}

In order to obtain some interesting limiting equations, we now make the assumption of maximal balance (the small terms are of the same order) $\varepsilon=\mu=\gamma^{2}$. This reflects a balance of weak nonlinearity and weak dispersion.

In this section, we study a Special Case, that is, when the waves velocities in the shallow water equations (78) and (79) are the same:

$$
\frac{\alpha_{1}}{\mu}(1-\tilde{\rho})=\frac{1-\rho}{\rho}=: c_{o}^{2} .
$$

Here below, we assume the following asymptotic expansions for $q, Q$, and $\widetilde{Q}$ :

$$
\begin{aligned}
q & =q_{0}+\varepsilon q_{1}+\cdots, \\
Q & =Q_{0}+\varepsilon Q_{1}+\cdots, \\
\widetilde{Q} & =\widetilde{Q}_{0}+\varepsilon \widetilde{Q}_{1}+\cdots .
\end{aligned}
$$

We also introduce new variables:

$$
\begin{aligned}
\xi=x_{1}-c_{0} t, & \zeta & =x_{1}+c_{0} t, \\
T=\varepsilon t, & y & =x_{2},
\end{aligned}
$$

which are travelling waves variables and describe the direction of waves propagation along the positive $x$-axis: $\xi$ represents right moving waves and $\zeta$ represents left moving waves.

In terms of the new variables, one obtains

$$
\partial t=-c_{0} \partial \xi+c_{0} \partial \zeta+\varepsilon \partial T ; \quad \partial x_{1}=\partial \xi+\partial \zeta .
$$

Considering the assumption of maximal balance together with (80), (79) becomes

$$
\begin{aligned}
q_{t t} & -c_{o}^{2} q_{x_{1} x_{1}}-\frac{\tilde{\rho}}{\alpha_{2}} \varepsilon\left[\widetilde{Q}_{t t}+q_{t t}\right]+\frac{\varepsilon}{2} \partial t\left(q_{x_{1}}{ }^{2}\right) \\
& -\varepsilon c_{0}^{2} q_{x_{2} x_{2}}+\varepsilon \partial x_{1}\left(q_{t} q_{x_{1}}\right)=0 .
\end{aligned}
$$


Substituting the expansions (81), (83) into (86) and equating L.O. terms yield the wave equations: $q_{0 t t}-q_{0 x x}=0$ and $\widetilde{Q}_{0 t t}-$ $\widetilde{Q}_{0 x x}=0$, whose solutions, respectively, are $q_{0}=F(\xi, T)+$ $G(\zeta, T)$ and $\widetilde{Q}_{0}=\widetilde{F}(\xi, T)+\widetilde{G}(\zeta, T)$.

We also assume unidirectional waves and only work with the right moving waves, so that

$$
\begin{aligned}
q_{0} & =F(\xi, T), \\
Q_{0} & =\widehat{F}(\xi, T), \\
\widetilde{Q}_{0} & =\widetilde{F}(\xi, T) .
\end{aligned}
$$

Then, keeping the $o(\varepsilon)$ terms, (86) becomes

$$
-4 c_{0}^{2} q_{1 \xi \zeta}=2 c_{0} F_{\xi T}+\frac{\tilde{\rho} c_{0}^{2}}{\alpha_{2}}\left[\widetilde{F}_{\xi \xi}+F_{\xi \xi}\right]+3 c_{0} F_{\xi} F_{\xi \xi}+c_{0}^{2} F_{y y} .
$$

In order to remove secular terms, the right-hand side of (88) needs to be set to zero (see [18]):

$$
2 c_{0} F_{\xi T}+\frac{\tilde{\rho} c_{0}^{2}}{\alpha_{2}}\left[\widetilde{F}_{\xi \xi}+F_{\xi \xi}\right]+3 c_{0} F_{\xi} F_{\xi \xi}+c_{0}^{2} F_{y y}=0 .
$$

Following the same steps as above for (78), together with (89), and studying only the $(1+1)$-dimensional case, we obtain a set of two equations:

$$
\begin{aligned}
& 2 c_{0} F_{\xi T}+\frac{\tilde{\rho} c_{0}^{2}}{\alpha_{2}}\left[\widetilde{F}_{\xi \xi}+F_{\xi \xi}\right]+3 c_{0} F_{\xi} F_{\xi \xi}=0, \\
& 2 c_{0} \widetilde{F}_{\xi T}-\frac{1}{\rho \widetilde{\rho}}\left(2 c_{0} F_{\xi T}+c_{0} F_{\xi} F_{\xi \xi}\right)+\frac{\alpha_{2} c_{0}^{2}}{\rho} F_{\xi \xi \xi \xi} \\
& +3 c_{0} \widetilde{F}_{\xi} \widetilde{F}_{\xi \xi}=0 .
\end{aligned}
$$

In the following, we will study only the $(1+1)$-dimensional case of the derivation.

Finally, define the functions $U=F_{\xi}, \widetilde{U}=\widetilde{F}_{\xi}$; then, differentiating (90) with respect to $\xi$, we can write the resulting equations in terms of $U$ and $\widetilde{U}$ as

$$
\begin{gathered}
2 U_{\xi T}+\frac{c_{0} \widetilde{\rho}}{\alpha_{2}}\left[\widetilde{U}_{\xi \xi}+U_{\xi \xi}\right]+\frac{3}{2} \partial_{\xi}^{2}\left(U^{2}\right)=0, \\
2 \widetilde{U}_{\xi T}-\frac{1}{\rho \tilde{\rho}}\left[2 U_{\xi T}+\frac{1}{2} \partial_{\xi}^{2}\left(U^{2}\right)\right]+\frac{\alpha_{2} c_{0}}{\rho} U_{\xi \xi \xi \xi}+\frac{3}{2} \partial_{\xi}^{2}\left(\widetilde{U}^{2}\right)=0 .
\end{gathered}
$$

Substitute (91) into (92) and obtain

$$
\begin{aligned}
& 2 \rho \widetilde{\rho} \widetilde{U}_{\xi T}+\frac{c_{0} \widetilde{\rho}}{\alpha_{2}} \widetilde{U}_{\xi \xi}+\frac{c_{0} \widetilde{\rho}}{\alpha_{2}} U_{\xi \xi}+\partial_{\xi}^{2}\left(U^{2}\right)+\alpha_{2} \widetilde{\rho} c_{0} U_{\xi \xi \xi \xi} \\
& +\frac{3}{2} \rho \widetilde{\rho} \partial_{\xi}^{2}\left(\widetilde{U}^{2}\right)=0 .
\end{aligned}
$$

Our multiple scale derivation leads therefore to having a system of two equations: (91) and (93) constitute a system of coupled nonlinear shallow water equations.

The two coupled shallow water equations will be studied numerically as a function of the parameters entering the theory, in order to prove the existence of solitary waves and analyze their behaviour.

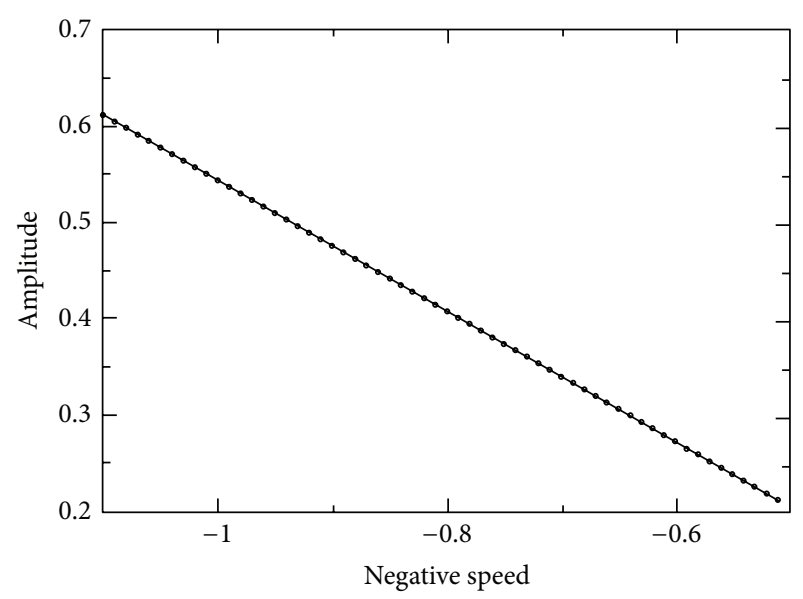

FIGURE 2: Amplitude versus negative speed for $\alpha_{2}=0.2, \mu=0.1$.

4.1. Numerical Investigation of Traveling Wave Solutions of the Coupled Shallow Water Equations. We now investigate whether (91) and (93) possess traveling wave solutions. For convenience, take $c_{0}=1$. Passing to a traveling coordinate system moving with velocity $c$ in the $\xi$ direction, (91) and (93) become, respectively,

$$
\begin{gathered}
-2 c U_{\xi \xi}+\frac{\tilde{\rho}}{\alpha_{2}}\left[\widetilde{U}_{\xi \xi}+U_{\xi \xi}\right]+\frac{3}{2} \partial_{\xi}^{2}\left(U^{2}\right)=0, \\
-2 c \rho \widetilde{\rho} \widetilde{U}_{\xi \xi}+\frac{\tilde{\rho}}{\alpha_{2}}\left[\widetilde{U}_{\xi \xi}+U_{\xi \xi}\right]+\alpha_{2} \tilde{\rho} U_{\xi \xi \xi \xi}+\partial_{\xi}^{2}\left(U^{2}\right) \\
+\frac{3}{2} \rho \widetilde{\rho} \partial_{\xi}^{2}\left(\widetilde{U}^{2}\right)=0 .
\end{gathered}
$$

To solve (94) numerically, we use the spectral renormalization (SPRZ) method, developed in [19]. We take the Fourier transform of the previous equations and get, upon rearranging,

$$
\begin{gathered}
\widehat{U}=\frac{2 c \rho \tilde{\rho} \widehat{\widetilde{U}}+(1 / 2) \widehat{U^{2}}-(3 / 2) \rho \tilde{\rho} \widehat{\widetilde{U}^{2}}}{2 c+\tilde{\rho} k_{1}^{2} \alpha_{2}}, \\
\widehat{\widetilde{U}}=\frac{-2 c \alpha_{2} \widehat{U}-\tilde{\rho} \widehat{U}+(3 / 2) \alpha_{2} \widehat{U^{2}}}{\tilde{\rho}} .
\end{gathered}
$$

In general, we cannot find a solution to (95)-(96) by naive iteration. Instead, we assume that $U=\lambda v$ and $\widetilde{U}=\widetilde{\lambda} \widetilde{v}$, where $\lambda, \tilde{\lambda}$ are unknown parameters and $v, \widetilde{v}$ are unknown functions (this step is the renormalization part). Then, (95) can be written in terms of $\lambda$ and $v$ as

$$
\widehat{v}=\frac{1}{\lambda}\left(\frac{2 c \rho \tilde{\rho}(\tilde{\lambda} \widehat{\widetilde{v}})+(1 / 2) \lambda^{2} \widehat{v^{2}}-(3 / 2) \rho \tilde{\rho}\left(\widetilde{\lambda}^{2} \widehat{\widetilde{v}^{2}}\right)}{2 c+\widetilde{\rho} k_{1}^{2} \alpha_{2}}\right) .
$$



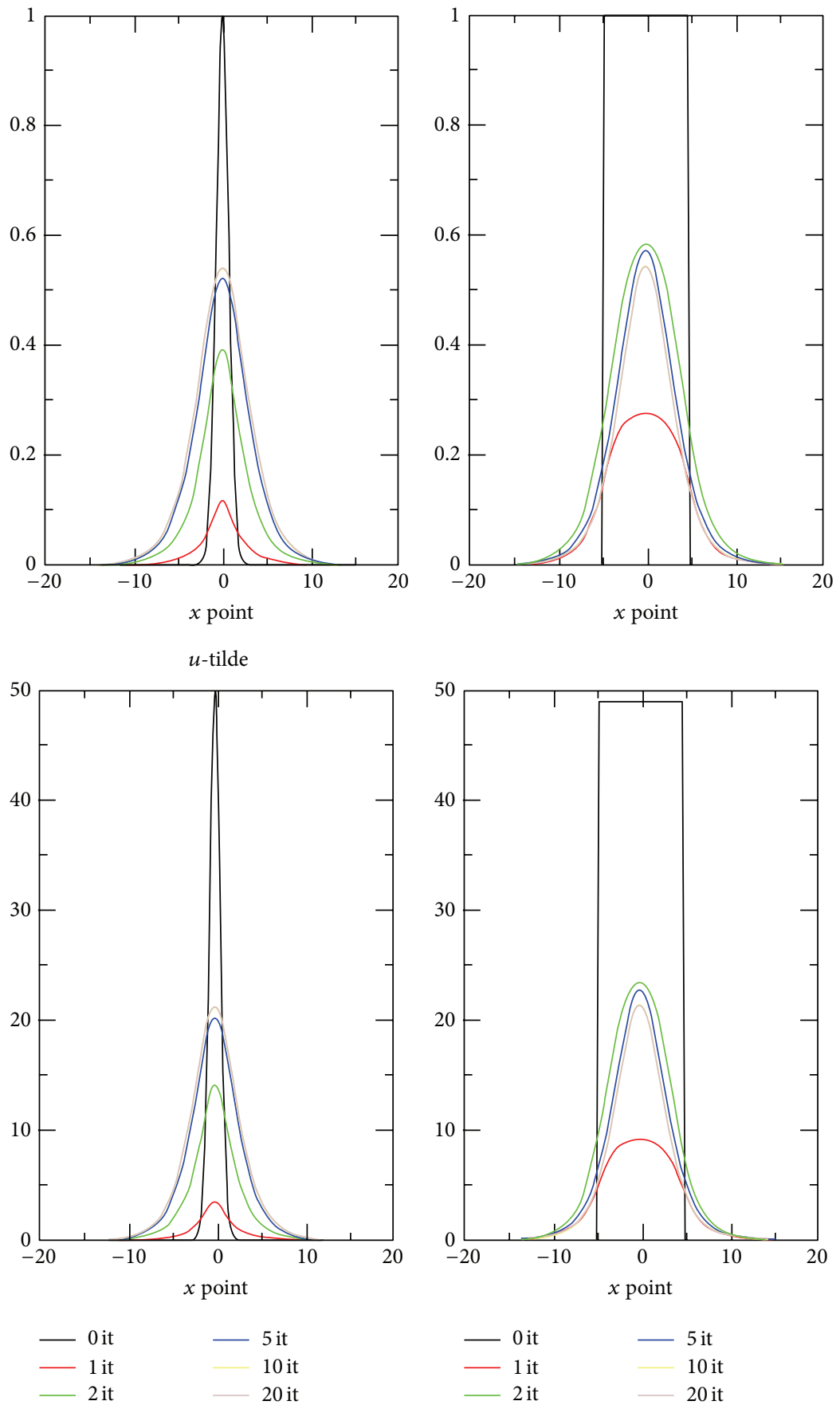

FIgURE 3: $x$ cross sections of $U$ and $\widetilde{U}$ for $\alpha_{2}=0.2, \mu=0.05, c=-1, \rho=0.5$, and $\widetilde{\rho}=0.7$.

Note that by multiplying (97) by $\overline{\hat{v}}$ (where $\overline{\hat{v}}$ denotes the conjugate of $\widehat{v}$ ), rearranging and integrating the result we get
Along the same line, (96) can be written in terms of $\widetilde{\lambda}$ and $\widetilde{v}$ as

$$
\lambda=\frac{\int_{\mathbb{R}}\left[2 c \rho \tilde{\rho}(\tilde{\lambda} \widetilde{\tilde{v}})+(1 / 2) \lambda^{2} \widehat{v}^{2}-(3 / 2) \rho \tilde{\rho}\left(\widetilde{\lambda}^{2} \widehat{\widetilde{v}}\right)\right] \overline{\hat{v}} d k_{1}}{\int_{\mathbb{R}}\left[2 c+\tilde{\rho} k_{1}^{2} \alpha_{2}\right] \widehat{\widehat{v}} d k_{1}} . \quad \widehat{\widetilde{v}}=\frac{1}{\tilde{\tilde{\lambda}}}\left(\frac{-2 c \alpha_{2}(\lambda \widehat{v})-\tilde{\rho}(\lambda \widehat{v})+(3 / 2) \alpha_{2}\left(\lambda^{2} \widehat{v^{2}}\right)}{\tilde{\rho}}\right) .
$$


By multiplying (99) by $\overline{\widehat{\widetilde{v}}}$ (where $\overline{\overline{\widetilde{v}}}$ denotes the conjugate of $\widehat{\widetilde{v}}$ ), rearranging and integrating the result we get

$$
\tilde{\lambda}=\frac{\int_{\mathbb{R}}\left[-2 c \alpha_{2}(\lambda \widehat{v})-\tilde{\rho}(\lambda \widehat{v})+(3 / 2) \alpha_{2}\left(\lambda^{2} \widehat{v^{2}}\right)\right] \overline{\widehat{\widehat{v}}} d k_{1}}{\int_{\mathbb{R}} \tilde{\rho} \overline{\widehat{\rho} \overline{\hat{v}}} d k_{1}} .
$$

We use the above SPRZ scheme to solve for the modes $U$ and $\widetilde{U}$ when $\alpha_{2}=0.2, c=-1, \rho=0.5$, and $\widetilde{\rho}=0.7$.

Figure 2 displays the resulting speed versus amplitude relationship, which is nearly linear for $\alpha_{2}=0.2$ (shallow water regime). Note that the horizontal axis in Figure 2 is $-c$.

Figure 3 shows the $x$ cross sections of $U$ and $\widetilde{U}$; on the left there are the solutions starting from a Gaussian function and on the right the ones starting from a step function, both for a typical speed $c=-1$ in the shallow water regime.

After obtaining these results, it would now be of particular interest from the applied point of view to address the issue of a local breakdown of the nonlinear internal waves propagating in the density stratified fluid [20]. Phenomena of this kind can induce localized turbulence in the stratified fluid and are relevant for their occurrence in the atmosphere and the oceans. We plan to address this issue in the future together with a generalization of our model to the $(2+1)$-dimensional case.

\section{Conflict of Interests}

The authors declare that there is no conflict of interests regarding the publication of this paper.

\section{Acknowledgment}

The authors wish to thank M. J. Ablowitz for useful suggestions.

\section{References}

[1] R. R. Long, "Some aspects of the flow of stratified fluids: I. A theoretical investigation," Tellus, vol. 5, no. 1, pp. 42-58, 1953.

[2] R. R. Long, "Some aspects of the flow stratified fluids. II. Experiments with a two-fluid system," Tellus, vol. 6, no. 2, 1954.

[3] J. T. Lin and Y. H. Pao, "Wakes in stratified fluids," Annual Review of Fluid Mechanics, vol. 11, pp. 317-338, 1979.

[4] H. J. S. Fernando, "Turbulent mixing in stratified fluids," Annual Review of Fluid Mechanics, vol. 23, no. 1, pp. 455-493, 1991.

[5] K. B. Winters, P. N. Lombard, J. J. Riley, and E. A. D'Asaro, "Available potential energy and mixing in density-stratified fluids," Journal of Fluid Mechanics, vol. 289, pp. 115-128, 1995.

[6] L. M. Milne-Thomson, Theoretical Hydrodynamics, Dover, New York, NY, USA, 1996.

[7] K. R. Helfrich and W. K. Melville, "Long nonlinear internal waves," Annual Review of Fluid Mechanics, vol. 38, pp. 395-425, 2006.

[8] T. B. Benjamin, "Internal waves of permanent form in fluids of great depth," Journal of Fluid Mechanics, vol. 29, no. 3, pp. 559592, 1967.
[9] H. Ono, "Algebraic solitary waves in stratified fluids," Journal of the Physical Society of Japan, vol. 39, no. 4, pp. 1082-1091, 1975.

[10] R. I. Joseph, "Solitary waves in a finite depth fluid," Journal of Physics A: Mathematical and General, vol. 10, no. 12, pp. 225227, 1977.

[11] T. Kubota, D. R. S. Ko, and L. D. Dobbs, "Weakly-nonlinear, long internal gravity waves in stratified fluids of finite depth," Journal of Hydronautics, vol. 12, no. 4, pp. 157-165, 1978.

[12] W. Choi and R. Camassa, "Fully nonlinear internal waves in a two-fluid system," Journal of Fluid Mechanics, vol. 396, pp. 1-36, 1999.

[13] W. Choi and R. Camassa, "Weakly nonlinear internal waves in a two-fluid system," Journal of Fluid Mechanics, vol. 313, pp. 83$103,1996$.

[14] W. Craig, D. Guyenne, and H. Kalisch, "Hamiltonian long-wave expansions for free surfaces and interfaces," Communications on Pure and Applied Mathematics, vol. 58, no. 12, pp. 1587-1641, 2005.

[15] M. J. Ablowitz, A. S. Fokas, and Z. H. Musslimani, "On a new non-local formulation of water waves," Journal of Fluid Mechanics, vol. 562, pp. 313-343, 2006.

[16] A. S. Fokas, "On the integrability of linear and nonlinear partial differential equations," Journal of Mathematical Physics, vol. 41, no. 6, pp. 4188-4237, 2000.

[17] T. S. Haut and M. J. Ablowitz, "A reformulation and applications of interfacial fluids with a free surface," Journal of Fluid Mechanics, vol. 631, pp. 375-396, 2009.

[18] M. J. Ablowitz, Nonlinear Dispersive Waves: Asymptotic Analysis and Solitons, Cambridge University Press, Cambridge, UK, 2011.

[19] M. J. Ablowitz and Z. H. Musslimani, "Spectral renormalization method for computing self-localized solutions to nonlinear systems," Optics Letters, vol. 30, no. 16, pp. 2140-2142, 2005.

[20] E. J. Hopfinger, “Turbulence in stratified fluids: a review, Journal of Geophysical Research: Oceans, vol. 92, no. 5, pp. 5287-5303, 1987. 


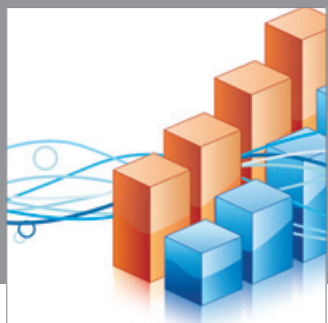

Advances in

Operations Research

mansans

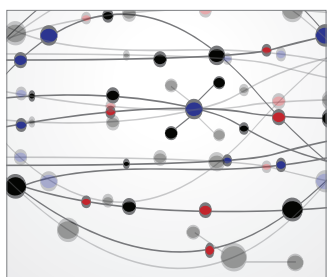

The Scientific World Journal
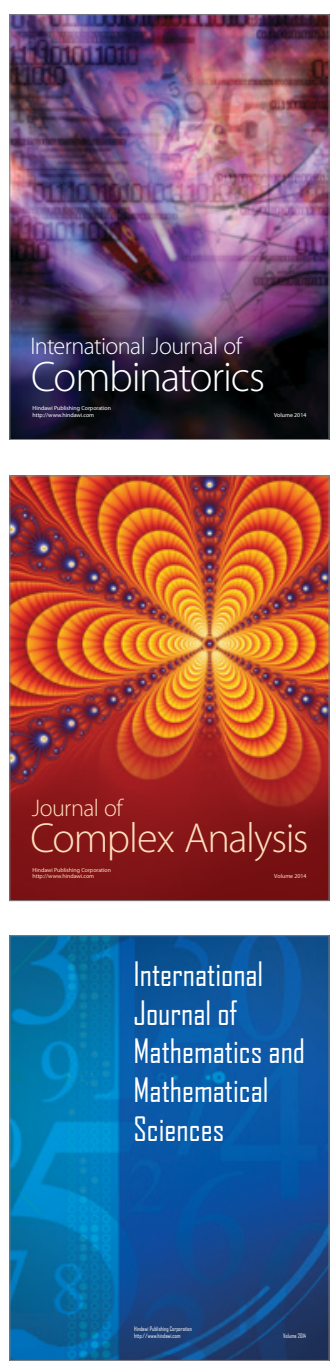
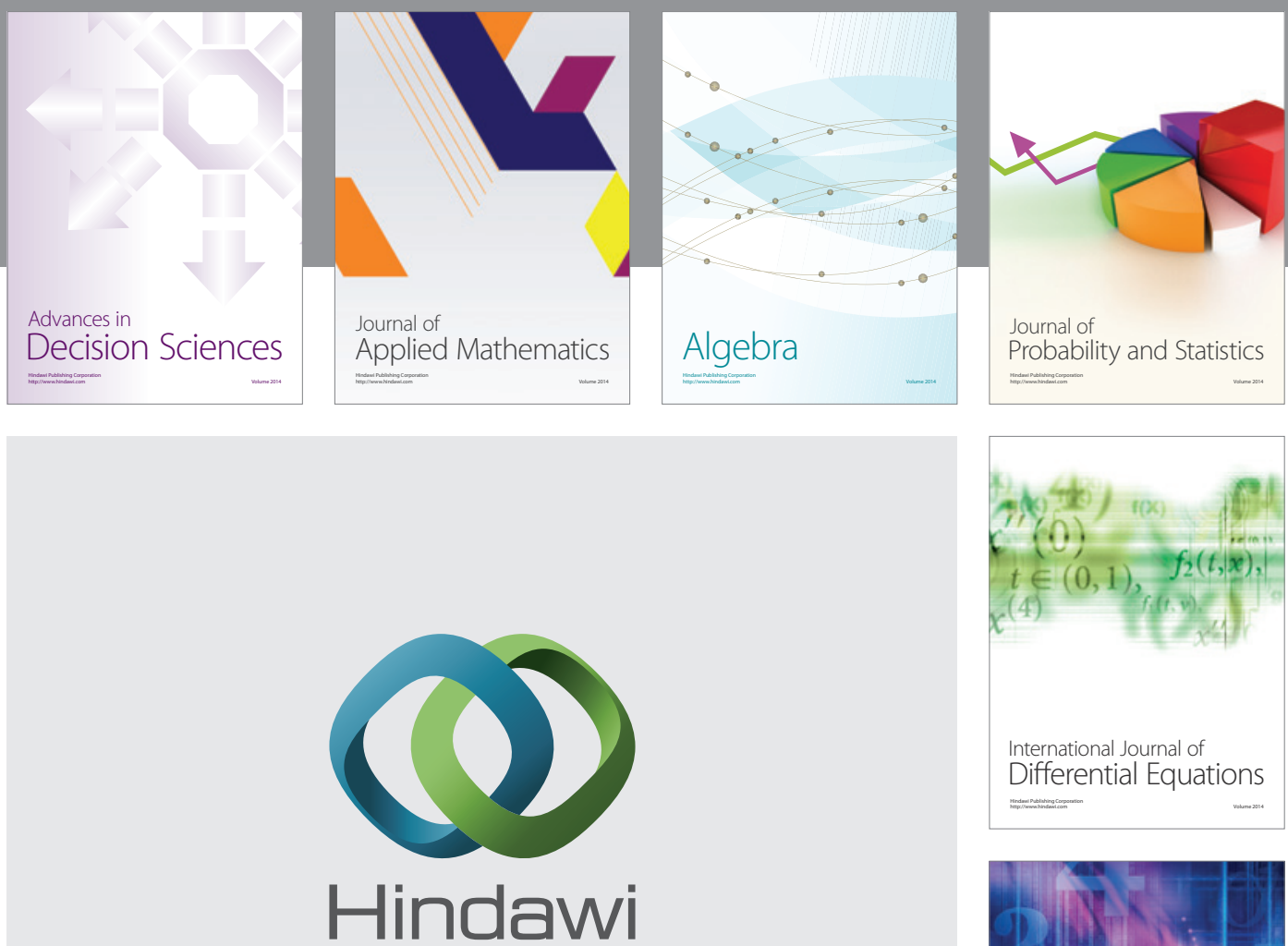

Submit your manuscripts at http://www.hindawi.com
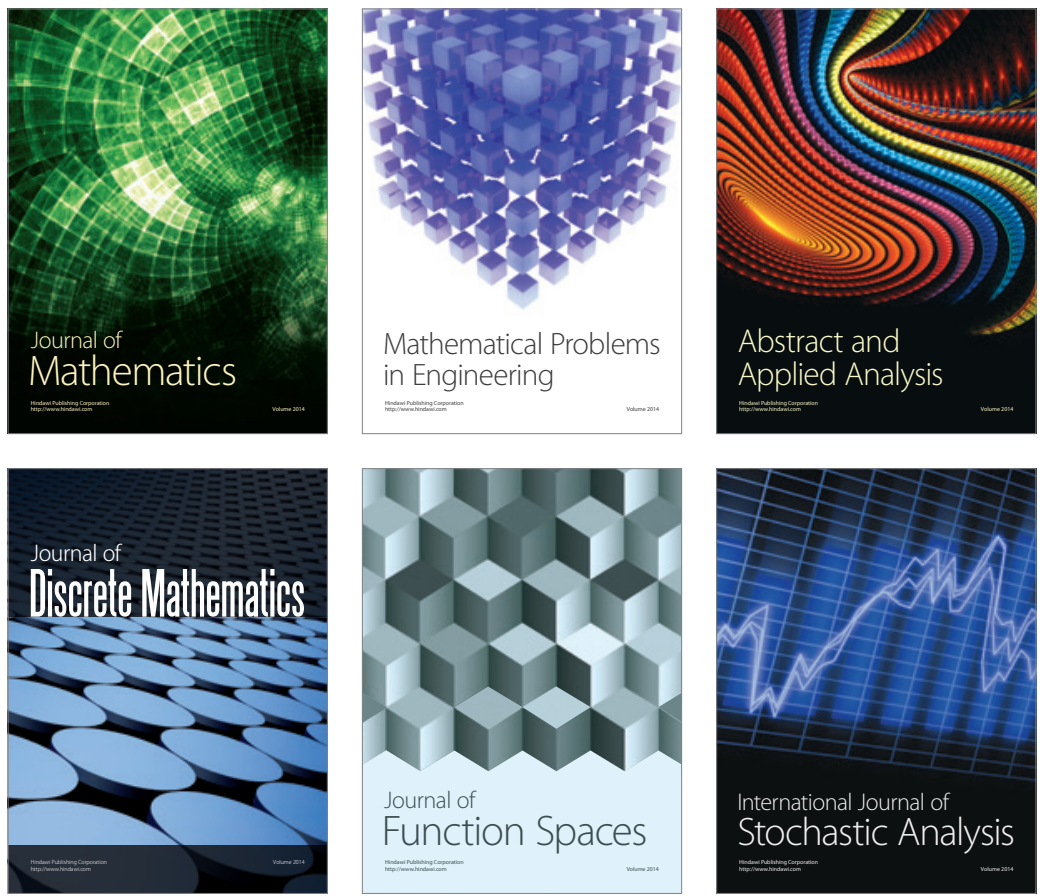

Journal of

Function Spaces

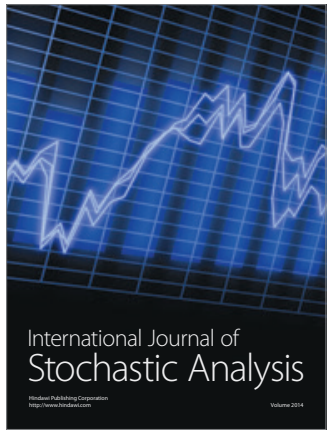

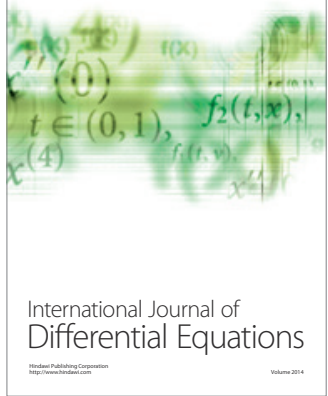
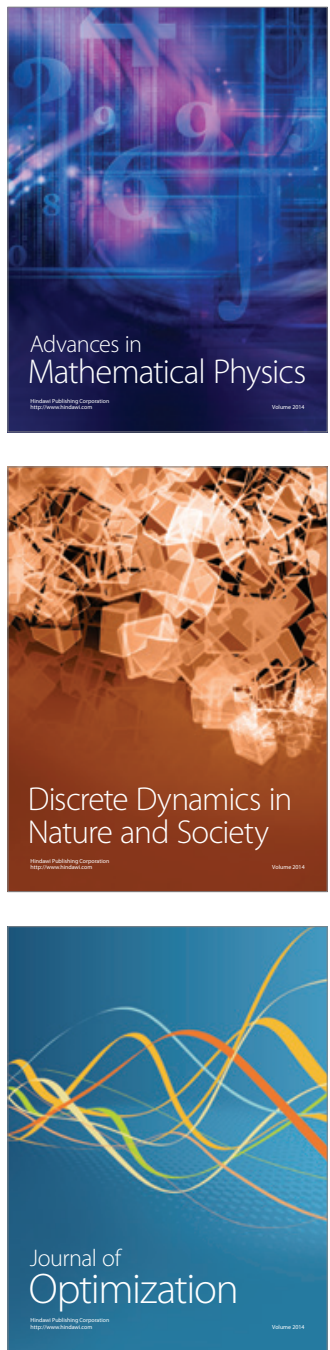